\title{
High Serum Thyrotropin Concentrations within the Reference Range: A Predictor of Malignancy in Nodular Thyroid Disease
}

\author{
Karla Duccini ${ }^{a}$ Marcus Vinicius Leitão de Souza ${ }^{a}$ Ricardo Delfim ${ }^{a}$ \\ Ana Paula Aguiar ${ }^{b}$ Patricia Teixeira ${ }^{a}$ Mario Vaisman ${ }^{a}$ \\ a Division of Medicine, Department of Endocrinology, Hospital Universitário Clementino Fraga Filho, \\ Rio de Janeiro, Brazil; b Division of Medicine, Department of Cytopathology, Hospital Universitário Clementino \\ Fraga Filho, Federal University of Rio de Janeiro, Rio de Janeiro, Brazil
}

\section{Significance of the Study}

- In this study, higher levels of thyrotropin, even within the normal range, indicated a higher risk of differentiated thyroid cancer among patients subjected to fine-needle aspiration biopsy. In practice, when higher levels of thyrotropin are present, it is preferable to perform fine-needle aspiration biopsy on sonographically indeterminate nodules with sizes around the threshold for biopsy, while surgical treatment is favored for indeterminate cytological biopsies.

\section{Keywords}

Thyroid nodule · Differentiated thyroid cancer · Thyrotropin · Risk factor · Bethesda classification

\footnotetext{
Abstract

Objective: This study assessed whether or not a simple biochemical parameter, serum thyrotropin (TSH), within the reference range can predict the likelihood of thyroid malignancy in subjects undergoing fine-needle aspiration biopsy (FNAB) for thyroid nodules. Subjects and Methods: This cross-sectional study evaluated 236 patients without overt thyroid dysfunction who were not on levothyroxine therapy. They underwent FNAB of the thyroid nodules at the Clementino Fraga Filho University Hospital between 2011 and 2012.
}

The reference range and serum TSH quartiles were determined according to age-specific values for serum TSH and then compared between the cases with benign thyroid nodular disease and differentiated thyroid carcinoma (DTC) based on surgical pathology reports. Results: Of the 236 patients, 201 (85.1\%) underwent a surgical procedure (thyroidectomy) and 89/236 (44.3\%) had DTC. The mean TSH concentrations were $2.09 \pm 1.15 \mathrm{mU} / \mathrm{L}$ in benign patients versus $2.73 \pm 1.33 \mathrm{mU} / \mathrm{L}$ in cases of DTC $(p<0.01)$. As TSH increased from quartiles 1 to 4 , the frequency of a malignant diagnosis also increased progressively from $22 \%$ in quartile 1 to $65 \%$ in the last quartile $(p \leq 0.001)$. Conclusion: Thyrotropin levels were positively associated with the diagnosis of DTC in thyroid nodules.

(c) 2018 The Author(s)

Published by S. Karger AG, Basel

\begin{tabular}{ll}
\hline KARGER & $\begin{array}{l}\text { @ 2018 The Author(s) } \\
\text { Published by S. Karger AG, Basel }\end{array}$ \\
$\begin{array}{l}\text { Openger } \\
\text { E-Mail karger@karger.com }\end{array}$ & $\begin{array}{l}\text { Thisis an Open Access article licensed under the Creative Commons } \\
\text { Attribution-NonCommercial-4.0 International License (CC BY-NC) } \\
\text { (hwtp://www.karger.com/Services/OpenAccessLicense), applicable to } \\
\text { the online version of the article only. Usage and distribution for } \\
\text { commercial purposes requires written permission. }\end{array}$
\end{tabular}

Marcus Vinicius Leitão de Souza

Hospital Universitário Clementino Fraga Filho, Universidade Federal do Rio de Janeiro Rua Prof. Rodolpho Paulo Rocco 255, 90 andar sala 9E23

Rio de Janeiro, RJ 21941-913 (Brazil)

E-Mail marcusleitao@ hucff.ufrj.br 


\section{Introduction}

Thyroid cancer is the most common endocrine malignancy, and its prevalence continues to rise [1]. Differentiated thyroid carcinoma (DTC) accounts for $80-90 \%$ of all tumors diagnosed, and it most often occurs as papillary carcinoma followed by follicular thyroid carcinoma [2, 3]. Fine-needle aspiration biopsy (FNAB) is the main diagnostic procedure for distinguishing benign from malignant thyroid disease. This method is the most cost-effective for determining the need for further interventions [4]. The challenge of evaluating a thyroid nodule is to exclude cancer $[3,4]$.

In recent studies, it was suggested that a high concentration of thyroid-stimulating hormone (TSH) within the normal range is associated with a diagnosis of thyroid cancer in patients with thyroid nodules [5-7]. Furthermore, this high level of TSH within the normal range was associated with advanced stages of thyroid cancer [8]. These findings indicated that TSH could play a central role in the development or progression of thyroid carcinomas to a well-established growth factor for thyroid nodules $[8,9]$. Equally important is that the use of suppressive doses of thyroxine $\left(\mathrm{T}_{4}\right)$ has been recognized to positively affect the outcome in treating DTC $[9,10]$. Retrospective studies have shown that the degree of TSH suppression is a prognostic determinant of DTC outcome [11-13].

Based on these findings, we postulated that the higher rates of malignancy found in patients with high TSH concentrations reflect a tropic effect of TSH on thyroid tissue and contribute to tumor development and carcinogenesis. Hence, the aim of the present study was to evaluate whether or not high TSH levels within the normal reference range can be used as a predictor of malignancy in nodular thyroid disease.

\section{Subjects and Methods}

\section{Subjects}

This cross-sectional study reviewed data from 236 patients (208 females and 28 males) who underwent FNAB of thyroid nodules at the Clementino Fraga Filho University Hospital between 2011 and 2012. At the time of FNAB, all patients were clinically and biochemically euthyroid (defined as normal serum concentrations of free $\mathrm{T}_{4}\left[\mathrm{fT}_{4}\right]$ and $\mathrm{TSH}$ ). Of the 236 patients subjected to FNAB, 201 (85.1\%) underwent surgery. A final histological diagnosis was obtained for all patients who underwent surgery.

The exclusion criteria were a past or present history of thyroid disease, previous thyroid surgery, use of medicines known to possibly interfere with analytical or physiological TSH or $\mathrm{fT}_{4}$ measure- ments within the past 3 months (medicines and contrast agents containing iodine in the past 6 months) [14-18], the use of antidepressants, and pregnancy. All of the patients selected for this study were previously physically examined, assessed by thyroid ultrasonography (US), and underwent FNAB before the surgical procedure.

Thyroidectomy was performed for patients with malignant, suspicious, or repetitive indeterminate nodules according to the FNAB results. Surgery was indicated for benign disease when local symptoms were present or for aesthetic reasons. The study was approved by the Research Ethics Committee of the Clementino Fraga Filho University Hospital, Federal University of Rio de Janeiro.

\section{Serum Studies}

Serum fasting TSH and $\mathrm{fT}_{4}$ levels were obtained at our institution and measured within 3 months of FNAB using electrochemiluminescence immunoassays. Serum TSH concentrations were measured by an immunometric method. The reference intervals of TSH were specific for each age group based on recent studies, which showed that TSH increases progressively and significantly with age [19-24]. The reference intervals for each age group were as follows: $6-14$ years: $0.3-4.2 \mathrm{mU} / \mathrm{L} ; 15-60$ years: $0.3-4.3 \mathrm{mUL}$; 61-79 years: $0.4-5.8 \mathrm{mU} / \mathrm{L}$; > 80 years: $0.4-6.7 \mathrm{mU} / \mathrm{L}$.

The reference intervals for TSH in each age group were divided into quartiles of similar size; we then combined the equivalent quartiles. The goal was to demonstrate the prevalence of malignancy (DTC) in the different quartiles in relation to the TSH values adjusted for age. The serum $\mathrm{fT}_{4}$ concentration was measured by competitive assays. The reference interval provided by the manufacturer is $0.9-1.7 \mathrm{ng} / \mathrm{dL}$.

\section{Thyroid FNAB and Pathology}

FNAB was performed for thyroid nodules $>1$ or $<1 \mathrm{~cm}$ with suspicious US features. Cytological findings were classified according to the Bethesda system as follows: nondiagnostic or unsatisfactory (Bethesda I); benign (Bethesda II); atypical or follicular lesion of undetermined significance (Bethesda III); follicular neoplasm or suspicious for a follicular neoplasm (Bethesda IV); suspicious for malignancy (Bethesda V); and malignant (Bethesda VI).

The Bethesda System for Reporting Thyroid Cytopathology (TBSRTC) recommends using 1 of 6 general diagnostic categories for reporting thyroid FNA results. The categories stratify the risk of malignancy and are associated with clinical management guidelines [25-27]. The final histological results following resection were available for all patients who underwent surgery $(n=201)$. An experienced pathologist reviewed all of the results to confirm the presence or absence of DTC.

\section{Statistical Analysis}

The main diagnostic outcome was defined as the presence or absence of malignancy (thyroid cancer) based on histopathology. The evaluation of the Bethesda categories was also included in the analysis. The influence of different factors on the final diagnostic outcome was investigated statistically, including age, gender, and serum TSH concentration. The survey of the prevalence of malignancy in the different TSH quartiles enabled judgments to be made on the validity of including TSH as a continuous variable.

All of the continuous variables were expressed as the median \pm $\mathrm{SD}$ or median and interquartile range (25th-75th percentiles). 
Table 1. Frequencies of thyroid-stimulating hormone (TSH) quartiles among each of the Bethesda categories

\begin{tabular}{|c|c|c|c|c|c|c|}
\hline TSH & Bethesda I & Bethesda II & Bethesda III & Bethesda IV & Bethesda V & Bethesda VI \\
\hline Quartile 1 & (27) $46.6 \%$ & (19) $22.1 \%$ & (4) $20.0 \%$ & (0) $0.0 \%$ & (6) $13.1 \%$ & (4) $23.5 \%$ \\
\hline Quartile 2 & (19) $32.8 \%$ & (30) $34.9 \%$ & (8) $40.0 \%$ & (5) $55.6 \%$ & (18) $39.1 \%$ & (3) $17.6 \%$ \\
\hline Quartile 3 & (6) $10.3 \%$ & (27) $31.4 \%$ & (7) $35.0 \%$ & (3) $33.3 \%$ & (10) $21.7 \%$ & (3) $17.6 \%$ \\
\hline Quartile 4 & (6) $10.3 \%$ & (10) $11.6 \%$ & (1) $5.0 \%$ & (1) $11.1 \%$ & (12) $26.1 \%$ & (7) $41.3 \%$ \\
\hline
\end{tabular}

Bethesda system - cytological diagnostic category from fine-needle aspiration biopsy (FNAB) of thyroid nodules: Bethesda I, nondiagnostic or unsatisfactory; Bethesda II, benign; Bethesda III, atypia of indeterminate significance or follicular lesion of indeterminate significance; Bethesda IV, follicular neoplasm or suspicious for follicular neoplasm; Bethesda V, suspicious for malignancy; Bethesda VI, malignant. $p<0.001$ in the comparison of all categories.

These variables were compared between groups by the MannWhitney or Kruskal-Wallis tests. The Mann-Whitney test was also applied to compare ordinal variables between 2 groups. Categorical variables were expressed as proportions and compared between groups by $\chi^{2}$ test or Fisher exact test. The level of significance was set at 5\%. Statistical analysis was carried out using SPSS software.

\section{Results}

The mean age of the patients was $50.0 \pm 15.6$ (range 13-67) years for females and $52.6 \pm 10.4$ (range 67-79) years for males. The mean serum TSH of the patients was $2.32 \pm 1.27(0.3-5.8) \mu \mathrm{IU} / \mathrm{mL}$. The cytological findings of the 236 nodules biopsied were classified according to the Bethesda system. Among these, there were 58 (24.6\%) classified as Bethesda I, 86 (36.5\%) as Bethesda II, 20 (8.4\%) as Bethesda III, 9 (3.8\%) as Bethesda IV, 46 (19.5\%) as Bethesda V, and 17 (7.2\%) as Bethesda VI. In categories III, IV, V, and VI, $100 \%$ of the patients underwent surgery (Fig. 1).

Table 1 shows the frequencies of TSH quartiles among each group according to the Bethesda category of thyroid nodules, and Figure 2 shows the frequencies of the 3 most important Bethesda categories (benign, indeterminate, and suspect/malignant) according to each quartile. A minority of patients with benign categories had serum TSH concentrations in the highest quartile. A majority of patients with malignant cytology (Bethesda VI) had serum TSH concentrations at the highest levels (Table 1). As shown in Figure 2, the patients with the highest quartile had a higher frequency ( $p=0.003$ ) of suspicious or malignant cytology (Bethesda V or VI). When evaluating patients in the lowest quartile, a higher frequency of benign cytology (Bethesda II) was detected, but the difference was not statistically significant.

Serum Thyrotropin and Malignant Thyroid Nodule

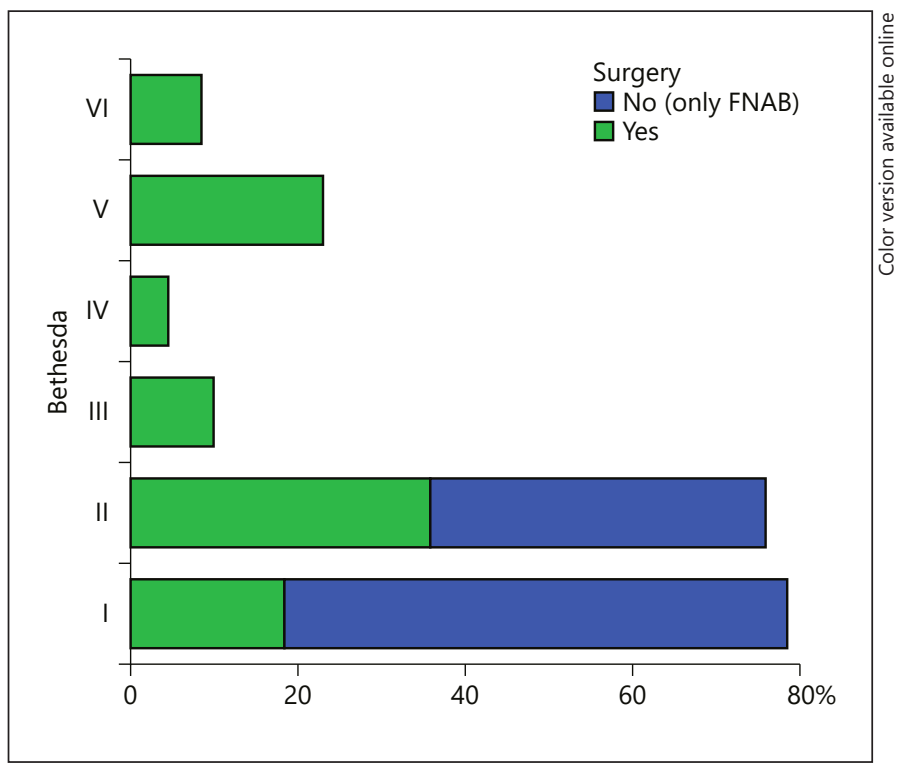

Fig. 1. Evaluated sample and frequency of thyroidectomy according to the Bethesda categories in the studied group. Bethesda system - cytological diagnostic category from fine-needle aspiration biopsy (FNAB) of thyroid nodules: Bethesda I, nondiagnostic or unsatisfactory; Bethesda II, benign; Bethesda III, atypia of indeterminate significance or follicular lesion of indeterminate significance; Bethesda IV, follicular neoplasm or suspicious for follicular neoplasm; Bethesda V, suspicious for malignancy; Bethesda VI, malignant.

Of the 201 patients who underwent surgery, 112 (55.72\%) were diagnosed with benign thyroid nodular disease, and the remaining $89(44.28 \%)$ had DTC. The median TSH concentrations were $1.90 \pm 1.15 \mathrm{mU} / \mathrm{L}$ in the 112 patients with no evidence of malignancy and $2.49 \pm$ $1.33 \mathrm{mU} / \mathrm{L}$ in the group of 89 patients with DTC (Fig. 3; $p<0.001)$. Analysis of the median TSH showed that there 


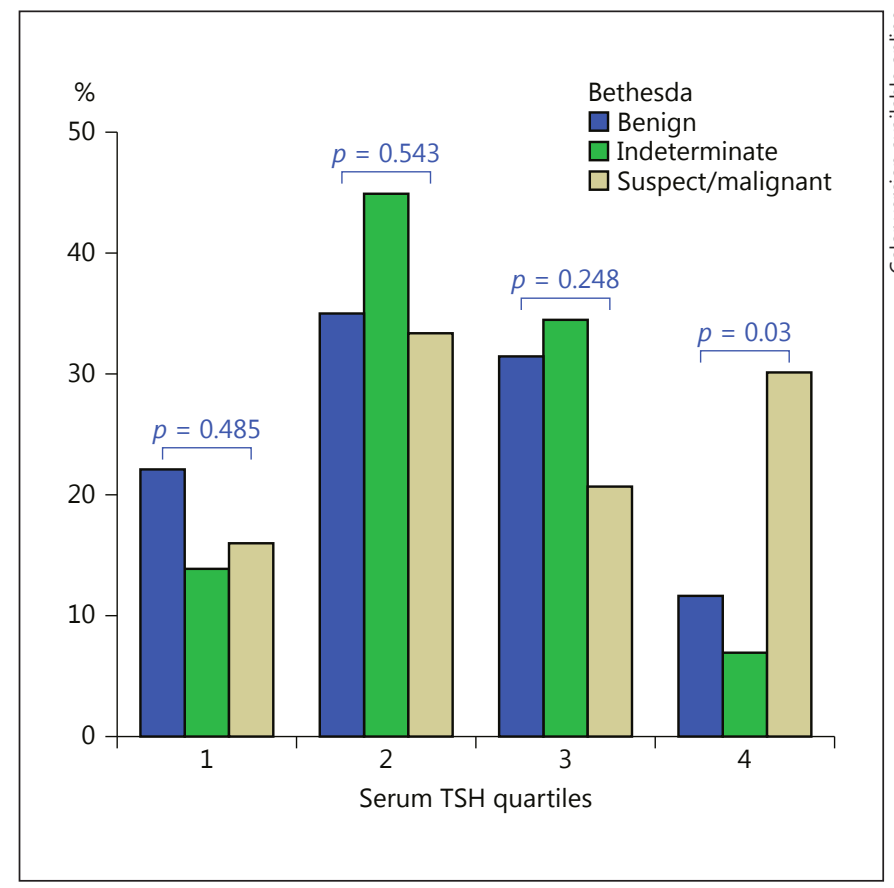

Fig. 2. Frequencies of the 3 most important Bethesda categories (benign, indeterminate, and suspect/malignant) according to each quartile ( $p<0.05$ in the comparison of the 3 groups; Kruskal-Wallis test). Comparing serum thyroid-stimulating hormone (TSH) quartiles, significance was only observed in quartile $4(p<0.005)$. TSH quartiles are age-adjusted quartiles of normal TSH. For further information, see legend to Figure 1.

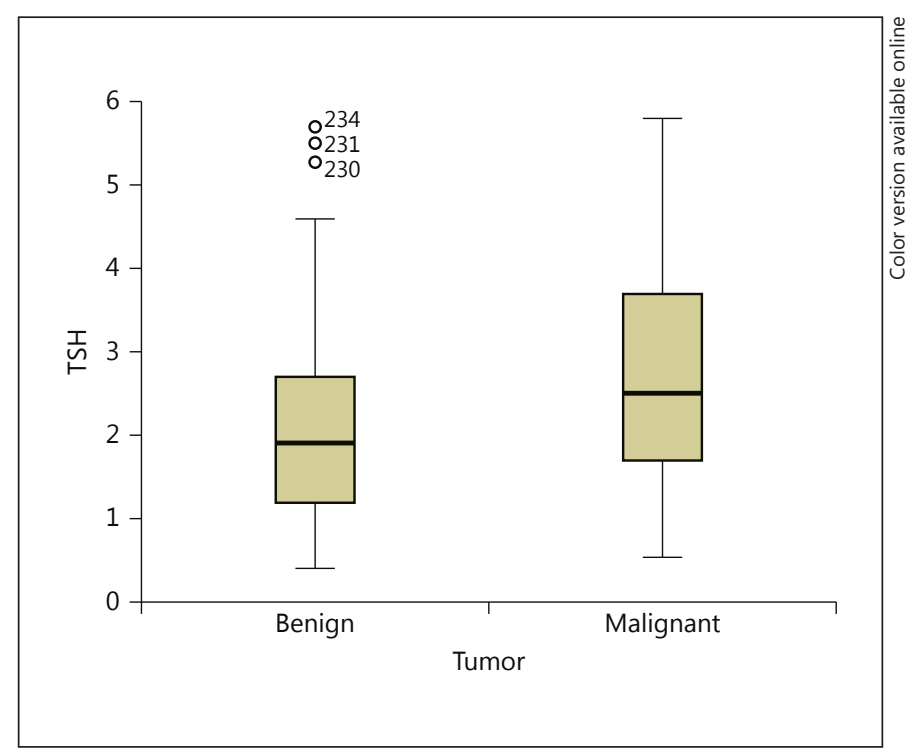

Fig. 3. Box plots showing serum thyroid-stimulating hormone (TSH) levels in the groups with benign and malignant disease. The top, bottom, and line through the box correspond to the 75th, 25th, and 50th percentiles (median), respectively $(p<0.001)$.

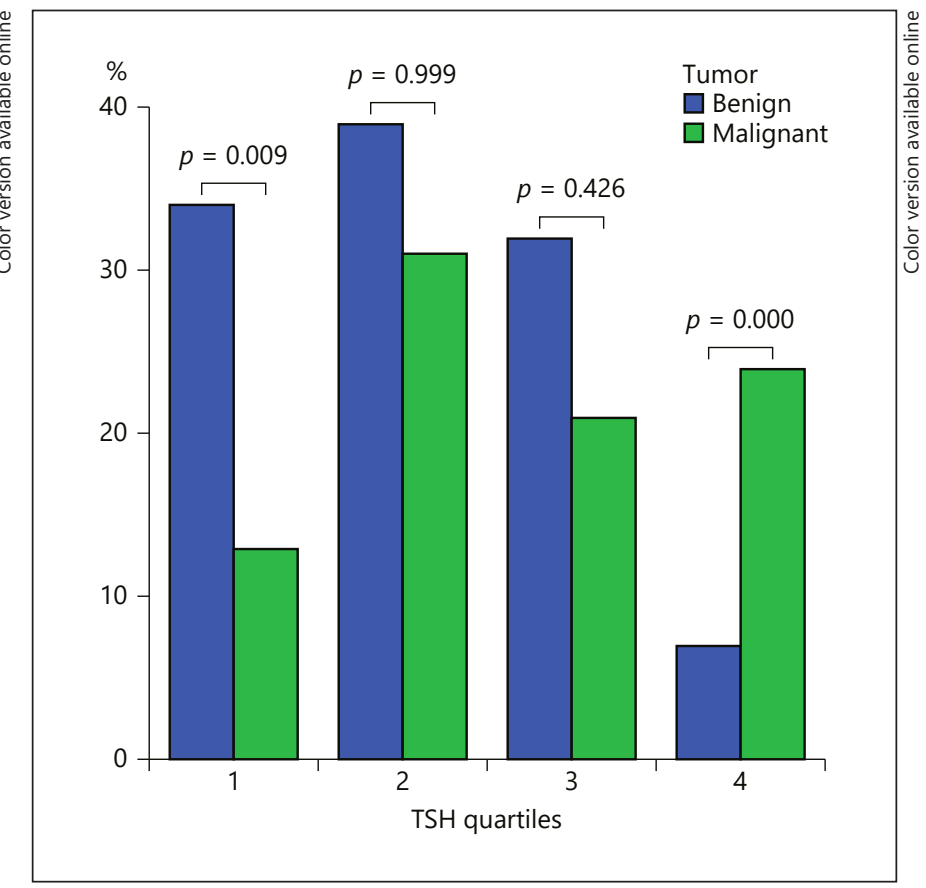

Fig. 4. Distribution of thyroid-stimulating hormone (TSH) levels in the normal range by quartiles adjusted for age in 2 histology groups. Kruskal-Wallis test. $\mathrm{p}<0.001$ in the comparison of all categories. were significantly higher TSH levels in the group with confirmed malignant disease (Fig. 3).

The patients with a serum TSH level within the normal range were divided into 4 quartiles of similar size according to their TSH values. The prevalence of DTC according to serum TSH increased in the highest quartile of serum $\mathrm{TSH}$, which indicates an increased prevalence of malignancy in those with higher TSH levels (Fig. 4).

\section{Discussion}

This is the first study to evaluate the association between quartiles of normal serum TSH with adjustment for age and the prevalence of differentiated thyroid carcinoma. Our results showed a statistically significant difference between the frequencies of malignancy among the highest quartile of TSH (65\%) compared to patients in the lowest quartile (22\%). These data are in accordance with previously published data [5-8], but we used quartiles adjusted for age, which is important since many recent 
studies show that TSH increases progressively and significantly with increasing age [20-24]. Thus, the TSH values were divided into quartiles of similar size in each age group, and equivalent quartiles were combined.

Boelaert et al. [5] were the first to report that serum TSH concentrations could be an independent predictor of thyroid malignancy in thyroid nodules that are investigated by FNA. They found that the risk of DTC diagnosis increases with serum TSH levels. Moreover, they derived a formula for the risk of malignancy based on the TSH level. However, we do not recommend this formula as in our study the cutoff point for each quartile was adjusted for age.

The hypothesis that TSH may be involved in the progression of DTC is supported by the observation that higher levels of TSH are associated with a more advanced stage of cancer, as reported by Haymart et al. [8]. In contrast, Shi et al. [28] reported that serum TSH is not a good risk predictor of differentiated thyroid microcarcinomas because an elevated TSH level may be related to an advanced stage of thyroid cancer progression but not with the development of thyroid cancer. Two reports demonstrated that a higher preoperative serum TSH was not only associated with the risk of DTC but also with a more advanced stage of cancer at the time of diagnosis $[8,29]$.

The role of the TSH level in the development and progression of thyroid cancer remains controversial. McLeod et al. [30] addressed this issue in a meta-analysis and suggested that there are no powerful prospective studies to support the idea that TSH induces thyroid cancer or that it promotes the growth of preexisting neoplasia. As our study is a cross-sectional analysis, we can only speculate about the positive association between TSH levels and the presence of DTC, which is confirmed by their meta-analysis, where a high TSH level within the normal range is suggested to be an unrecognized risk factor for DTC.

Jonklaas et al. [6] reported that this association remained in a strictly euthyroid population of 50 patients who underwent thyroid surgery for nodular thyroid disease (after excluding subclinical hypo- and hyperthyroidism cases). Polyzos et al. [7] confirmed that TSH levels are predictive of malignancy within a normal range only because they did not find this association in patients with subclinical hypothyroidism. As our sample had only euthyroid patients without any thyroxine replacement, the influence of subclinical dysfunction was not the object of our analysis.

Another hallmark is the evaluation of histopathology and not solely cytology. Few studies have attempted to integrate laboratory parameters systematically with the results of FNAB for the process of determining whether patients need surgery. Importantly, the diagnoses of DTC or benign thyroid disease in our sample were based on surgical pathological results, which increase the power of the association with DTC in our data.

Considering the measurement of serum TSH, which is a highly sensitive determinant of thyroid dysfunction, its evaluation is recommended as a biochemical test in the initial evaluation of patients presenting with thyroid nodules $[4,31]$. Our analysis demonstrates that the serum TSH concentration, even when it is within the normal range, can be used as an additional parameter to indicate a higher risk for the presence of thyroid malignancy when examining thyroid nodules. However, we could not determine any specific cutoff of TSH for the risk of DTC due to age adjustments for normal TSH values and the different quartiles that result. Nevertheless, an important practical implication, especially for general clinicians, may be the indication of cytological evaluation for nodules with sizes around the threshold for biopsy when TSH is high in the normal range. Equally important is the indication for surgery for an indeterminate cytology using higher normal TSH as another risk factor for malignancy.

One limitation of the present study is the absence of information about thyroid antibodies and the use of single TSH measurements. Notably, all patients had serum TSH levels in the respective reference range according to their age group.

\section{Conclusion}

In this study, a significant difference was confirmed between serum thyrotropin levels in patients with DTC and benign thyroid nodular disease. These observations could be relevant for the inclusion of thyroid function as a clinical parameter to be considered as a risk factor for DTC in patients with nodular thyroid diseases.

\footnotetext{
References $\quad>_{1}$ Davies L, Welch HG: Increasing incidence of thyroid cancer in the United States, 19732002. JAMA 2006;295:2164-2167.

2 Ries LAG, Harkins D, Krapcho M, et al: SEER Cancer Statistics Review, 1975-2003. Bethesda, National Cancer Institute, 2006, http:// seer.cancer.gov/csr/1975_2003/.

$\checkmark 3$ Coeli CM, Brito AS, Barbosa FS, et al: Incidence and mortality from thyroid cancer in Brazil. Arq Bras Endocrinol Metabol 2005;49: 503-509.
} 
4 Cooper DS, Doherty GM, Haugen BR, et al: Management guidelines for patients with thyroid nodules and differentiated thyroid cancer. Thyroid 2006;16:1-33.

$>5$ Boelaert K, Horacek J, Holder RL, et al: Serum thyrotropin concentration as a novel predictor of malignancy in thyroid nodules investigated by fine-needle aspiration. J Clin Endocrinol Metab 2006;91:4295-4301.

6 Jonklaas J, Nsouli-Maktabi H, Soldin SJ: Endogenous thyrotropin and triiodothyronine concentrations in individuals with thyroid cancer. Thyroid 2008;18:943-952.

7 Polyzos SA, Kita M, Efstathiadou Z, et al: Serum thyrotropin concentration as a biochemical predictor of thyroid malignancy in patients presenting with thyroid nodules. J Cancer Res Clin Oncol 2008;134:953-960.

>8 Haymart MR, Repplinger DJ, Leverson GE, et al: Higher serum thyroid stimulating hormone level in thyroid nodule patients is associated with greater risks of differentiated thyroid cancer and advanced tumor stage. J Clin Endocrinol Metab 2008;93:809-814.

-9 Papini E, Petrucci L, Guglielmi R, et al: Longterm changes in nodular goiter: a 5 -year prospective randomized trial of levothyroxine suppressive therapy for benign cold thyroid nodules. J Clin Endocrinol Metab 1998;83: $780-783$.

10 Mazzaferri EL, Jhiang SM: Long-term impact of initial surgical and medical therapy on papillary and follicular thyroid cancer. Am J Med 1994;97:418-428.

11 Pujol P, Daures JP, Nsakala N, et al: Degree of thyrotropin suppression as a prognostic determinant in differentiated thyroid cancer. J Clin Endocrinol Metab 1996;81:4318-4323.

$>12$ Jonklaas J, Sarlis NJ, Litofsky D, et al: Outcomes of patients with differentiated thyroid carcinoma following initial therapy. Thyroid 2006;16:1229-1242.
13 Hovens GC, Stokkel MP, Kievit J, et al: Associations of serum thyrotropin concentrations with recurrence and death in differentiated thyroid cancer. J Clin Endocrinol Metab 2007; 92:2610-2615.

14 Larkin JG, Macphee GJA, Beastall GH, et al: Thyroid hormone concentrations in epileptic patients. Eur J Clin Pharmacol 1989;36:213216.

15 Davies PH, Franklyn JA: The effects of drugs on tests of thyroid function. Eur J Clin Pharmacol 1991;40:439-451.

16 Stockigt JR: Free thyroid hormone measurement: a critical appraisal. Endocrinol Metab Clin North Am 2001;30:265-289.

17 Steele BW, Wang E, Klee GG, et al: Analytic bias of thyroid function tests: analysis of a College of American Pathologists fresh frozen serum pool by 3900 clinical laboratories. Arch Pathol Lab Med 2005;129:310-317.

18 Garber JR, Cobin RH, Gharib H, et al: Clinical practice guidelines for hypothyroidism in adults: cosponsored by the American Association of Clinical Endocrinologists and the American Thyroid Association. Thyroid 2012;22:1200-1235.

19 Fontes R, Coeli CR, Aguiar F, et al: Reference interval of thyroid stimulating hormone and free thyroxine in a reference population over 60 years old and in very old subjects (over 80 years): comparison to young subjects. Thyroid Res 2013;3:13-20.

20 Hollowell JG, Staehling NW, Flanders WD, et al: Serum TSH, $\mathrm{T}_{4}$, and thyroid antibodies in the United States population (1988-1994): National Health and Nutrition Examination Survey (NHANES III). J Clin Endocrinol Metab 2002;87:489-499.

21 Ceriotti F: Prerequisites for use of common reference intervals. Clin Biochem Rev 2007; 28:115-121.

22 Horowitz GL, Altaie S, Boyd JC, et al: CLSI C28-A3: Defining, Establishing, and Verifying Reference Intervals in the Clinical Laboratory; Approved Guideline, ed 3. Wayne, Clinical and Laboratory Standards Institute, 2008.
23 Kahapola-Arachchige KM, Hadlow N, Ward$\operatorname{rop} \mathrm{R}$, et al: Age-specific TSH reference ranges have minimal impact on the diagnosis of thyroid dysfunction. Clin Endocrinol 2012; 77:773-779.

24 Vadiveloo T, Donnan PT, Murphy MJ, et al: Age- and gender-specific TSH reference intervals in people with no obvious thyroid disease in Tayside, Scotland: the Thyroid Epidemiology, Audit, and Research Study (TEARS). J Clin Endocrinol Metab 2013;98:1147-1153.

25 Theoharis CG, Schofield KM, Hammers L, et al: The Bethesda thyroid fine needle aspiration classification system: year 1 at an academic institution. Thyroid 2009;19:12151223.

26 Cibas ES, Ali SZ: The Bethesda system for reporting thyroid cytopathology. Thyroid 2009; 19:1159-1165.

-27 Bongiovanni M, Spitale A, Faquin WC, et al: The Bethesda System for Reporting Thyroid Cytopathology: a meta-analysis. Acta Cytol 2012;56:333-339.

28 Shi L, Li Y, Guan H, et al: Usefulness of serum thyrotropin for risk prediction of differentiated thyroid cancers does not apply to microcarcinomas: results of 1,870 Chinese patients with thyroid nodules. Endocr J 2012;59:973980 .

29 Fiore E, Rago T, Provenzale MA, et al: Lower levels of TSH are associated with a lower risk of papillary thyroid cancer in patients with thyroid nodular disease: thyroid autonomy may play a protective role. Endocr Relat Cancer 2009;16:1251-1260.

30 McLeod DS, Watters KF, Carpenter AD, et al: Thyrotropin and thyroid cancer diagnosis: a systematic review and dose-response metaanalysis. J Clin Endocrinol Metab 2012;97: 2682-2692.

31 Harris PE: British Thyroid Association and Royal College of Physicians Guidelines for the management of thyroid cancer in adults. Clin Med (Lond) 2002;2:144-146. 